\title{
Creating digital models of paleoanthrological sample by photogrammetry and computed tomography
}

\author{
Novikov, Mikhail \\ FSRC \\ "Crystallography and \\ Photonics" RAS \\ Svyatoozerskaya, 1. Russia, \\ 140700, Shatura \\ novikov@rambler.ru
}

\author{
Knyaz, Vladimir \\ State Research Institute \\ of Aviation System \\ (GosNIIAS) \\ 7, Victorenko str., \\ Russia, 125319, Moscow \\ knyaz@gosniias.ru
}

\author{
Galeev,Ravil \\ Institute of Ethnology and \\ Anthropology RAS \\ Leninskiy prospect $32 \mathrm{a}$, \\ Russia, 119991, Moscow \\ ravil.galeev@gmail.com
}

\begin{abstract}
The specificity of paleoanthropological researchs and accurate anthropological documentation is due to the complexity of the form of anthropological objects, their uniqueness, high historical and scientific value. The main purpose of this work is to develop methods for creating 3D models with a high degree of informatively for the development of digital documentation systems of paleoanthropological objects. The article presents a comparative analysis of digital models of anthropological object create using photogrammetry and computed tomography. It is shown that the combined use of non-contact methods of photogrammetry and computed tomography allows to create high-precision three-dimensional models with photorealistic texture and accurate internal and hidden geometry. The proposed approach allows not only to create virtual collections for wide sharing of specialists, but also using modern methods of additive manufacturing to make exact copies of unique artifacts.
\end{abstract}

\section{Keywords}

paleoanthropology; non-contact measurements; digital model; computed tomography; photogrammetry; additive technologies; three-dimensional documentation

\section{INTRODUCTION}

One of the main objectives of paleoanthropology is the morphological description of bone remains, using a number of measuring methods (craniometry, osteometry, etc.), allowing for extensive comparative studies, the purpose of which is to describe the diversity of anthropological types, both in ancient times and in modern times [Jur16a].

Despite the significant development of modern measuring equipment, hand tools are used in modern physical anthropology. In part, this is due to the fact that the developed measuring techniques of paleoanthropological objects can be used with a specific set of measurement instrument - stout, jig, sliding compass and other tools (Figure 1), in addition, the accuracy of manual measurements is in full compliance with the required accuracy (the measurement accuracy is up to $0.1 \mathrm{~cm}$ ).

At the same time, manual measurement has a number of obvious shortcomings - it is, first of all, the complexity, time-consuming, often damage to the object of study, and, surprisingly, the extreme high cost of anthropological tools.

Modern non-contact methods of measurement allow to obtain huge arrays of geometric data of paleontological samples and to create on their basis high-precision

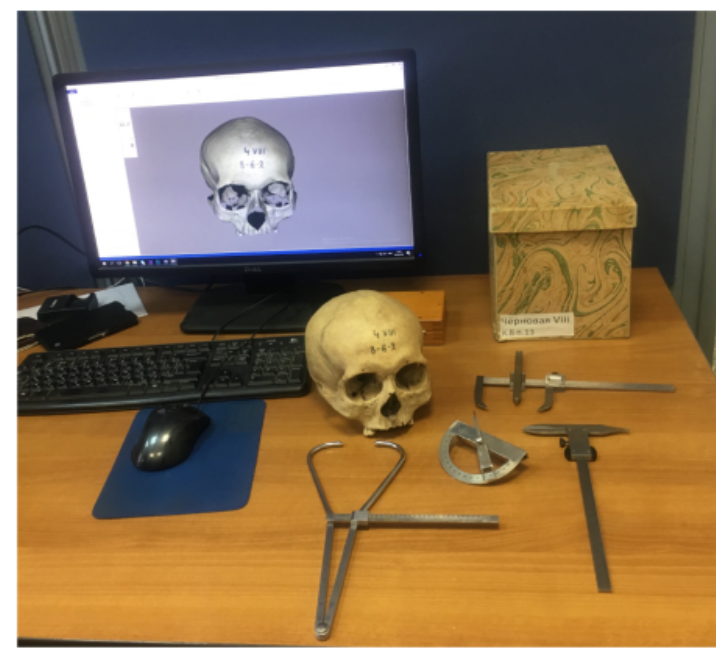

Figure 1: Mechanical tools for measuring anthropological objects

digital models for documenting and providing wide remote access to these data for specialists [Jur16a]. This is largely able not only to solve the above problems of manual measurement and eliminate their shortcomings, but also to expand the creative research horizons in general.

Paleoanthropology imposes special requirements on the created digital models [Web15], [Dav17]. Together with precision and the desired level of detail of the 
paleoanthropological models must be exactly are textured with high resolution. In addition, it is often necessary to use digital models with different levels of detail in different parts of the object [Sub02].

In geometric morphometry, the change in shape, defined as the spatial distribution of benchmark, can be analyzed independently of size using a set of morphometric features. The coordinates of the labels can be obtained either by using the digitizer directly from the object, or on digital models created by computer tomography (CT) [Nys15] or surface scanning [Heg16]. Computer tomography also offers the possibility of measuring internal structures.

Anthropology has a long history of measuring biological form. Currently, there are several applications that provide the ability to include new 3D benchmarks and geometric morphometry methods in the study of changes in size and shape. This approach allows for original research [Cun14].

\section{PHOTOGRAMMETRY}

The paleoanthropological material for this study was obtained as a result of the excavation of Chernovaya VIII, carried out in 1962-1963. G.P. Maksimenkov, and dates back to II millennium BC. [Mak80]. More than 50 skulls suitable for research were found in 14 burial mounds of the burial ground. For studying the paleoanthropology of the Okunev culture, the collected series of skulls is one of the most representative in terms of both the number and the preservation of anthropological material [Rav16].

Textured surface 3D models of paleoanthropological objects were obtained using an original photogrammetric system developed in the State Research Institute of Aviation System [Kny16]. For purposes of paleoanthropological analysis 3D models of paleoanthropological objects have to be of high geometric accuracy and of high resolution (detailing). Additionally some special requirements are imposed for the $3 \mathrm{D}$ reconstruction system. They are as following:

- Short processing time

- Producing 3D model of a given object

- Texturing of the 3D model to capture visual features of scanning area

To meet these requirements the system configuration shown in Figure 2 is chosen.

It includes two high-resolution digital cameras, a computer-controlled table for rotating the object (optional), a special structured light projector to illuminate the sample, and a digital SLR camera for high-resolution texture generation. Original software supports in automated a set of the functions needed for creating accurate photorealistic textured 3D models.
The main functions are:

- Automatic system calibration based on coded targets

- Scanning 2.5D fragments of an object

- Merging all partial 2.5D patches in single 3D mesh

- Accurate automatic mapping of the high resolution texture

The developed system is based on photogrammetric principle of spatial measurements, which allows determining 3D coordinates for any point of the object if its image correspondence for two oriented photographs is established. The system provides automated images orientation (calibration) and supports a set of methods of automated correspondence problem solution based on various structured light patterns such as scanning stripe, coded light, phase shift.

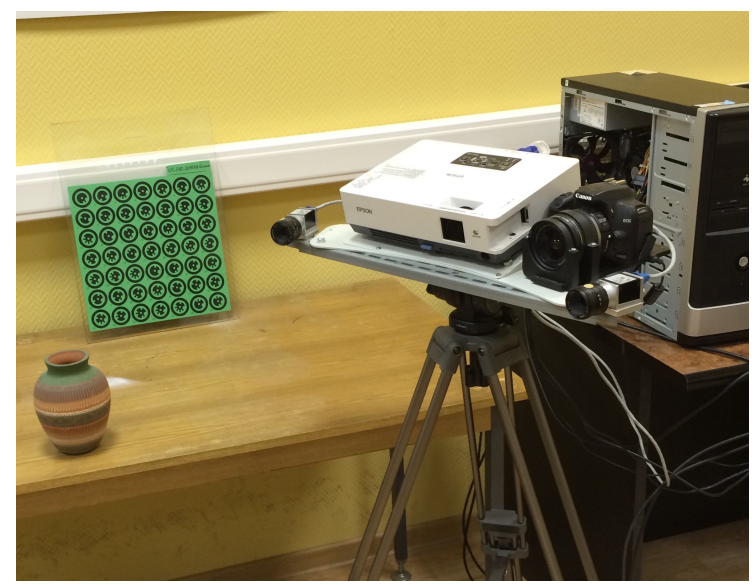

Figure 2: Photogrammetric system

The original calibration procedure [Kny10] is used to compensate geometrical distortions of imaging and to provide high accuracy of generated $3 \mathrm{D}$ models. It starts from the basic model of image formation - the collinearity equation, expressing the condition that the point of the scene $G$, the center of the projection of $O$ and the image of this point $g$ lie on one straight line:

$$
\mathbf{X}_{G}=\mathbf{X}_{0}-\mu \mathbf{A}^{T} \cdot\left(\mathbf{x}_{g}-\mathbf{x}_{p}\right)
$$

Here

$\mathbf{X}_{0}=\left(X_{0}, Y_{0}, Z_{0}\right)-$ coordinates of the center of the projection,

$\mathbf{X}_{G}=(X, Y, Z)-$ scene point coordinates,

$\mathbf{x}_{g}=(x, y,-f)-$ the corresponding coordinates of the scene point in the image,

A - coordinate system transformation matrix,

$\mathbf{x}_{p}$ - coordinates of the main point of the snapshot,

$\mu-$ scale factor.

In a real imaging system, an image is formed with the distortions introduced by the elements of the optical 
system of the lens and inaccuracies in the manufacture of the camera. To account for distortions, additional terms are introduced into the collinearity equations $\Delta x$ and $\Delta y$, describing various distorting factors. Then the real (distorted) $x_{d}, y_{d}$ coordinates of a point in the image are defined as:

$$
\begin{aligned}
& x_{d}=x+\Delta x ; \\
& y_{d}=y+\Delta y ;
\end{aligned}
$$

The practice of photogrammetric measurements have proved the good description for nonlinear distortion is the following model [Bey92]:

$$
\begin{aligned}
& \Delta x=a_{0} \cdot y_{a}+x_{a}\left(a_{1} r^{2}+a_{2} r^{4}+a_{3} r^{6}\right) \\
& +a_{4}\left(r^{2}+2 x_{a}^{2}\right)+2 a_{5} x_{a} y_{a} ; \\
& \Delta y=a_{0} \cdot x_{a}+y_{a}\left(a_{1} r^{2}+a_{2} r^{4}+a_{3} r^{6}\right) \\
& +a_{5}\left(r^{2}+2 y_{a}^{2}\right)+2 a_{4} x_{a} y_{a} ;
\end{aligned}
$$

here $r^{2}=x_{a}^{2}+y_{a}^{2}$

Here

$x_{a}, y_{a}$ - coordinates of a point on the image, $a_{0}, \ldots, a_{5}$ - camera interior orientation parameters: $a_{0}$ - coefficient of affine distortion;

$a_{1}, a_{2}, a_{3}$ - coefficients of radial distortion; $a_{4}, a_{5}$ - coefficients of tangential distortion.

Firstly laboratory calibration was performed using a precise test field. The test field contains 49 reference points with known 3D coordinates. The reference points are marked by coded targets providing automated detection, identification and precise sub-pixel measurement of reference points in the image. The vector of estimated parameters $v_{e}^{l}=\left(x_{p}, y_{p}, m_{x}, m_{y}, a_{0}, \ldots, a_{5}\right)^{T}$ for test field calibration includes coordinates of principal point, image scales

\begin{tabular}{|c|c|c|c|}
\hline \multicolumn{4}{|c|}{ Camera rmse, $\mathrm{mm}$} \\
\hline & Left & Right & Color \\
\hline$\sigma_{x}$ & 0.022 & 0.024 & 0.014 \\
\hline$\sigma_{y}$ & 0.023 & 0.021 & 0.011 \\
\hline$\sigma_{z}$ & 0.033 & 0.035 & 0.020 \\
\hline \multicolumn{4}{|c|}{ Max error, $\mathrm{mm}$} \\
\hline$\delta$ & 0.083 & 0.078 & 0.044 \\
\hline
\end{tabular}
and additional parameters correspondingly, spatial coordinates of reference points being known by independent precise measurements.

The results of calibration procedure are presented in Table 1.

Table 1: Results of system calibration
Table 1 ensures the accuracy of the generated 3D models at the level of $0.05 \mathrm{~mm}$. This accuracy is sufficient for the task of anthropological analysis.

The textured digital model provides the expert with more information, as some features can only be found on the color image of the object. Since the photogrammetric system has been calibrated for all three cameras using a single calibration field, this ensures that the texture is accurately superimposed on the geometric coordinates of the digital model.

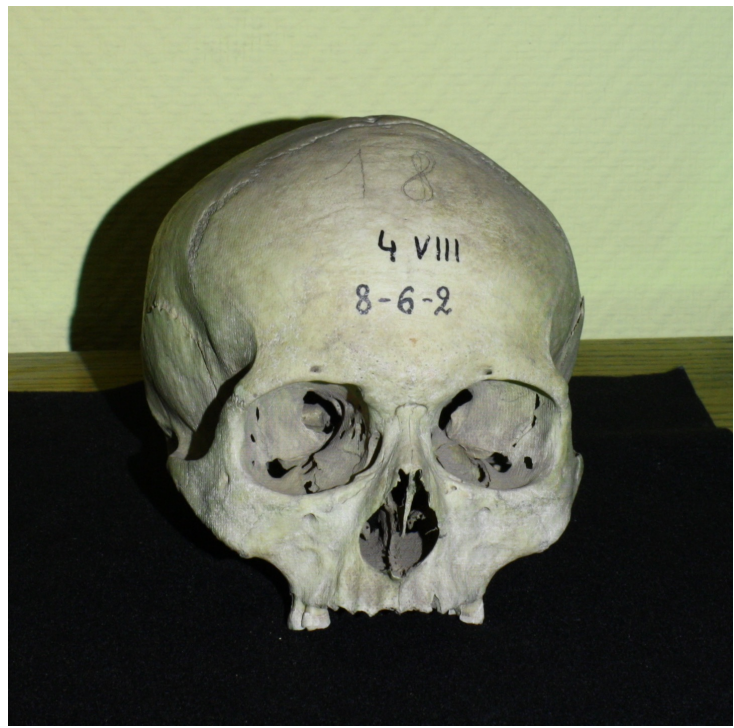

Figure 3: Object image

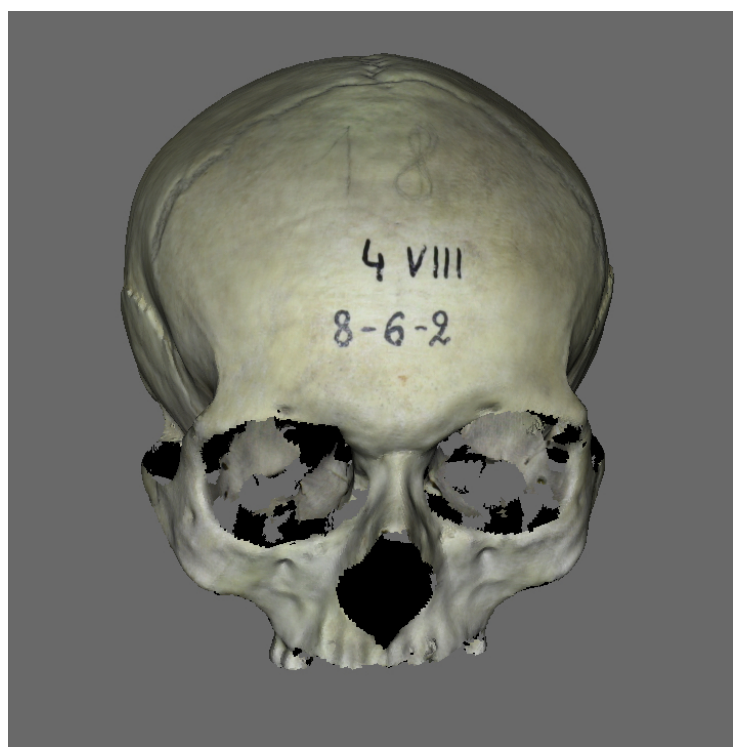

Figure 4: Object textured 3D model

Figure 3 presents an image of a skull. Figure 4 presents textured 3D model acquired with high resolution ("micro" configuration of the photogrammetric system).

These models can be used for paleoanthropological research and solving such problems as: 
- measurements of morphometric anthropological parameters (distances, angles, coordinates));

- virtual and plastic reconstruction of the external appearance;

- restoration of paleoanthropological objects-digital and manual;

- creation and documentation of specialized databases of paleoanthropological objects.

As an alternative to the traditional method, a set of algorithms for automatic detection and recognition of landmarks is proposed. They use both an object digital model and high-resolution textures to recognize a set of necessary landmarks.

\section{COMPUTER TOMOGRAPHY}

The study of the paleontological object was carried out on a modern multispiral computed tomography. The data obtained in DICOM [DICOM] format (433 slices with $0.572 \mathrm{~mm}$ step and $0.5723 \mathrm{~mm} \mathrm{X}$ and Y resolution) were transmitted over the network to ILIT RAS for processing and conversion into a three-dimensional digital model.

Tomograms are a set of gray images of arrays of sections (layers) of the object under study at the $z$ coordinate. Each element of the tomogram is a function of the density of the object at the corresponding point $q(x, y, z)$. To convert the tomogram into a digital model, it is necessary to build a mathematical model of the object as a solid. In general, such a model can be represented as:

$$
F(x, y, z)= \begin{cases}0 & : \text { no object } \\ 1 & \text { object present }\end{cases}
$$

the function $F\left(x_{i}, y_{j}, z_{k}\right)$ has the form of a threedimensional image with two gradations ( 0 or 1 - bits per pixel). Thus, if you represent an image pixel as a cube with dimensions $d x \times d y \times d z$, the transformation of tomographic data to a digital model can be performed by converting $Q(i, j, k)$ to $F\left(x_{i}, y_{j}, z_{k}\right)$.

For the conversion to be correct, it is necessary to correctly determine the boundary of the real object in the tomogram. In the simplest case, the ratio (1) takes the form

$$
F\left(x_{i}, y_{j}, z_{k}\right)= \begin{cases}0 & \text { if } Q(i, j, k) \leq Q_{t} \\ 1 & Q(i, j, k)>Q_{t}\end{cases}
$$

The correct definition of the object boundary is possible only if you know all the nuances of the mechanism of tomographic scanning of specific types of objects and representation of their images on the tomogram. After defining the boundaries of the object, the accuracy of the manufactured model is completely determined by the number of image points and the number of layers in the source data.

Tomographic data of the anthropological sample was processed in the programs Inobitec DICOM Viewer (Inobitec, Russia). Comparison of computer models is shown in (Figure 5).
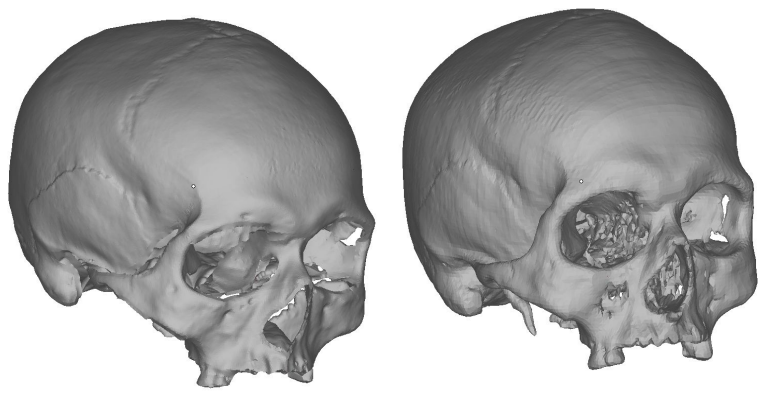

Figure 5: Left to right: anthropological model creating by photogrammetry and tomographic 3D model by programs Inobitec.

\section{TEXTURED THREE-DIMENSIONAL MODEL WITH INTERNAL STRUC- TURE}

The main objective of this work is to create a highprecision three-dimensional textured model of paleoanthropological sample. To do this, a combination of geometric data of the photogrammetric and tomographic models was carried out. Step-by-step combination of shift and rotation was carried out according to STL data in the program Magics (Materialise, Belgium). The results of this alignment and standard deviations are shown in Figure 6.
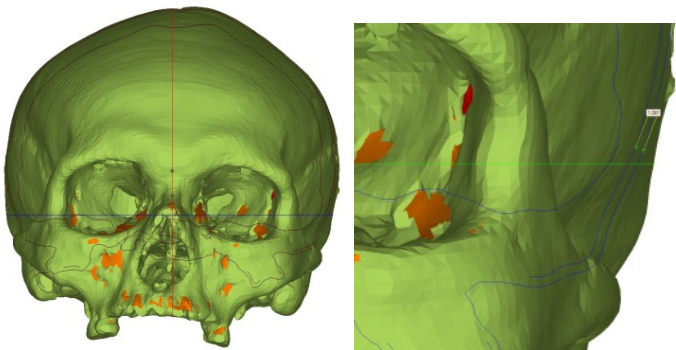

Figure 6: Step-by-step combination of STL files in the program Magics

The average deviation of the photogrammetric and tomographic models was $1.6 \mathrm{~mm}$.

In addition, the models were automatically combined in a specialized program for working with point data (Figure 7).

The obtained results showed a good coincidence of the geometric data created by photogrammetric and tomographic methods. This allows you to combine textured 


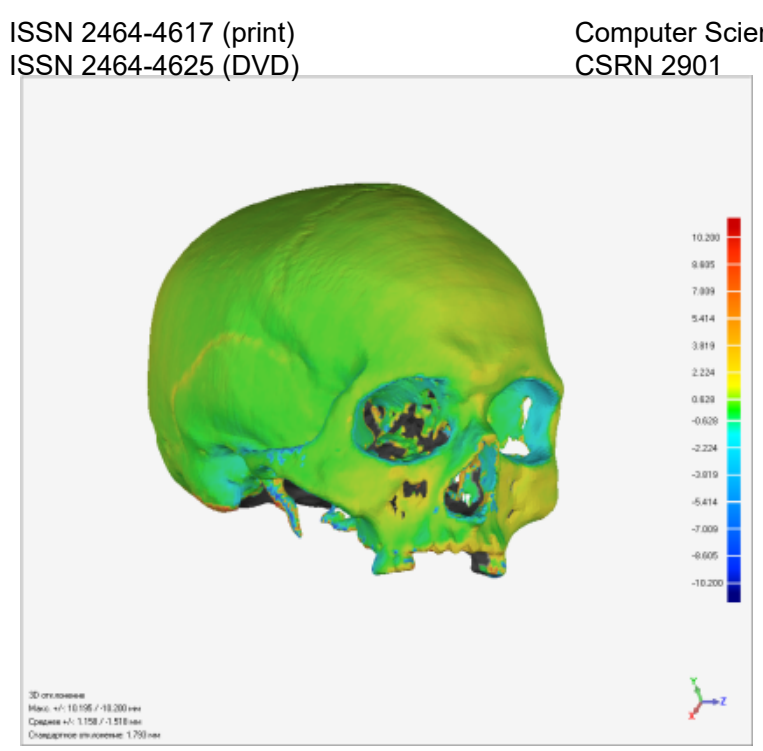

Figure 7: Automatic combination of photogrammetric and tomographic models by minimum mean deviation

geometrically bound data with the internal structure of the tomographic data and obtain a highly informative digital model (Figure 8).

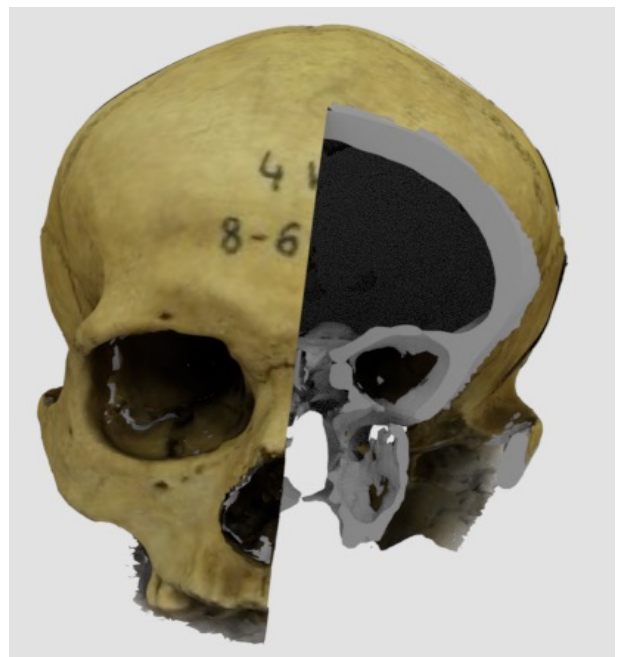

Figure 8: Combination of photogrammetric and tomographic models with high-resolution textures

\section{EXPERIMENTAL RESULTS. RE- CONSTRUCTION WITH THE USE OF ADDITIVE TECHNOLOGIES}

Another advantage of the introduction of digital models in the practice of paleoanthropological research is the possibility of obtaining their exact copies with the help of additive technologies. These copies of paleoanthropological objects can be used for artistic reconstruction of appearance, for creation of reference collections for wide access.

The digital model created by the photogrammetric system represents only the external surfaces of the object can not be directly used for reconstruction on 3D printers that use only solid-state models. The 3D surface model was converted into a solid model by setting an equidistant thickness in the Magics software.
WSCG Proceedings Part I This process required additional time and the resulting internal structure do not correspond to the real object.In the case of a digital model obtained by converting tomographic data, the entire structure of the object is reproduced in full, but information about the texture and appearance of the object is lost.

Plastic copies of paleoanthropological digital models were made on a laser stereolithograph LC250 [Che15], developed in ILIT RAS (Figure 9).

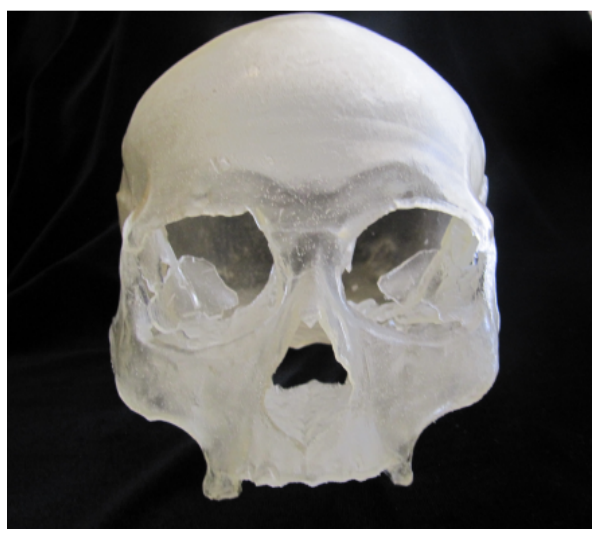

Figure 9: The result of the reconstruction of the paleontological object

The main purpose of creating real copies of digital models is to check the quality and accuracy of 3D printing and to study the possibility of using such copies in paleoanthropological studies. To assess the accuracy of the stereolithographic model it was scanned photogrammetric system. The resulting digital model was compared with the original model. The results of comparison of these two models are shown in (Figure 10).

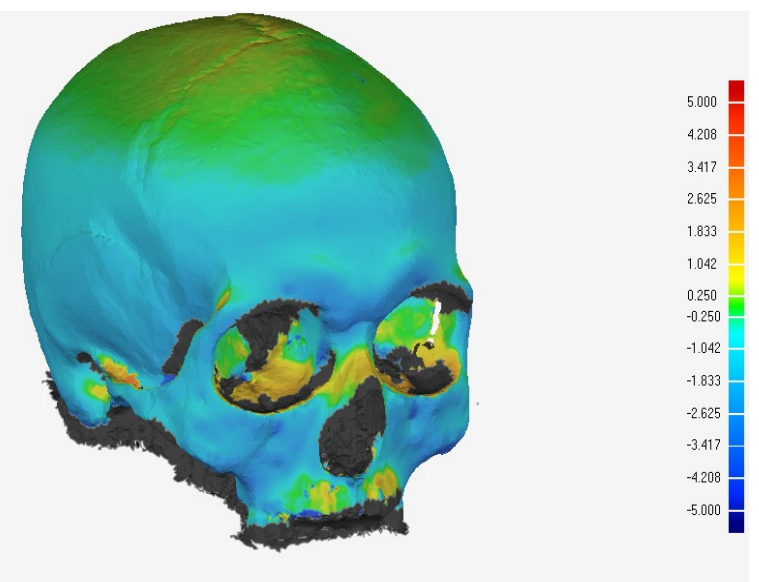

Figure10: The comparison results for two models

The results of estimates of the accuracy of stereolithography reconstruction show that SLA models can be used to solve such anthropological problems as reconstruction of human appearance and object restoration.

\section{CONCLUSION}

The problem of anthropological research and accurate documentation of the studied objects is associated with 
the complexity of their geometric shape, including the presence of hidden from view areas, as well as the fragility of high - value paleoanthropological materials. In this regard, the possibility of measurements by contact methods and their accuracy are significantly limited. The methods and means of non-contact mea-surements developing today create high-precision dig-ital models of the studied objects, to provide measure-ments with high accuracy, as well as to create conditions for the preservation of the objects of research, which is of great importance when working with paleoanthropological materials. Methods of creating digital three-dimensional models of complex spatial form provide opportunities for automation of measurement processes and application of data mining methods. The resulting digital models are the basis for creating exact copies of unique objects of cultural heritage with the help of additive technologies necessary for visualization and training.

Photogrammetric methods of collection, processing and presentation of paleoanthropological data have been de-veloped. The main means of reconstruction of digital paleoanthropological 3D models is a photogrammetric system that provides accurate and photorealistic 3D and 2D data.

A study of the same anthropological object was carried out using computed tomography. A digital model based on tomographic data was constructed and compared with a photogrammetric model. The resulting difference was due to the calibration of the photogrammetric system can be compensated by a scale factor.

The applicability of the use of stereolithographic copies of paleoanthropological objects for research and exchange of rare data is studied. Evaluation of the accuracy of the stereolithographic copies shows that they can be successfully used for educational purposes and for the reconstruction of the appearance.

\section{CONFLICT OF INTEREST}

The authors declare that they have no conflict of interest.

\section{ACKNOWLEDGMENTS}

This work was done with the financial support of the RFBR grant OFI-m N 17-29-04509.

\section{REFERENCES}

[Bey92] Beyer, H., Advances in characterization and calibration of digital imaging systems. Int. Arch. Photogramm. Remote Sens. Spatial Inf. Sci. XXIX, pp. 545-555, 1992.

[Che15] Cherebylo S.A., Evseev A.V., Ippolitov E.V., Novikov M.M., Applying laser stereolithography in medicine. At: Modern laser information technology. Ed.: V. Ya. Panchenko, F.V.Lebedeva, M.: Interkontakt Nauka, 358-3736 2015.
[Cun14] John A. Cunningham, Imran A. Rahman, Stephan Lautenschlager, Emily J. Ray- field, and Philip C.J. Donoghue. A virtual world of paleontology. Trends in Ecology \& Evolution, 29(6):347 - 357, 2014.

[Dav17] Davies T.G. et al. Open data and dig-ital morphology, Proc. R. Soc. B 284:20170194 http://dx.doi.org/10.1098/rspb.2017.0194, 2017

[DICOM] Digital Imaging and Communications in Medicine (DICOM), National Electrical Manufacturers Association, Rosslyn, USA, https://www.dicomstandard.org/current/.

[Heg16] Hegna Thomas A., Johnson Robert E. , Preparation of fossil and osteological 3d-printable models from freely available CT-scan movies, Journal of Paleontological Techniques, Number 16, pp. 1-10, 2016.

[Jur16a] Mikolas Jurda and Petra Urbanova. Threedimensional documentation of dolni vestonice skeletal remains: can photogrammetry substitute laser scanning? Anthropologie vol. 54, no. 2, pp. $109-118,2016$.

[Jur16b] Mikolas Jurda and Petra Urbanova. Sex and ancestry assessment of Brazilian crania using semi-automatic mesh processing tools Legal Medicine 23, 34 - 43, 2016.

[Kny10] Knyaz V., "Multi-media Projector - Single Camera Photogrammetric System For Fast 3D Reconstruction," Int. Arch. Photogramm. Remote Sens. Spatial Inf. Sci. XXXVIII-5, 343-348 (2010)

[Kny16] Knyaz, V. A. and Chibunichev, A. G., "Photogrammetric techniques for road surface analysis," ISPRS - International Archives of the Photogrammetry, Remote Sensing and Spatial Information Sciences XLI-B5, 515-520 (2016).

[Mak80] Maksimenkov G.A. Burial ground Chernovaya VIII - reference monument of Okunev culture // Monuments Okunev culture. L., 1980. pp. 3-22

[Nys15] Nysjö,J., Malmberg,F., Sintorn,I., Nyström,I.: BoneSplit - A 3D Texture Painting Tool For Interactive Bone Separation in CT Images, Journal of WSCG Vol.23, No.2, p.157, (2015).

[Rav10] Galeev R.M. Craniotriganometric research of turns from a burial ground Chernovaya VIII, Bulletin of archeology, anthropology and ethnography. 2010. № 2 (13) 109.

[Sub02] Subsol Gerard et al. Three-Dimensional Imaging in Paleoanthropology and Prehistoric Archaeology, pages $37-45$. BAR International Series 1049, 2002.

[Web15] Gerhard W. Weber. Virtual Anthropology and Biomechanics, pages 937 - 968. Springer Berlin Heidelberg, Berlin, Heidelberg, 2015. 